\title{
Substrate melting during laser heating of nanoscale metal films
}

\author{
Francesc Font ${ }^{\mathrm{a}}$, Shahriar Afkhami ${ }^{\mathrm{b}}$, Lou Kondic ${ }^{\mathrm{b}}$ \\ ${ }^{a}$ Department of Mathematics and Statistics, McMaster University, Hamilton, ON, \\ Canada \\ ${ }^{b}$ Department of Mathematical Sciences, New Jersey Institute of Technology, Newark, NJ, \\ USA
}

\begin{abstract}
We investigate heat transfer mechanisms relevant to metal films of nanoscale thickness deposited on a silicon ( $\mathrm{Si}$ ) substrate coated by a silicon oxide $\left(\mathrm{SiO}_{2}\right)$ layer and exposed to laser irradiation. Such a setup is commonly used in the experiments exploring self and directed assembly of metal films that melt when irradiated by laser and then evolve on time scale measured in nanoseconds. We show that in a common experimental setting, not only the metal but also the $\mathrm{SiO}_{2}$ layer may melt. Our study of the effect of the laser parameters, including energy density and pulse duration, shows that melting of the substrate occurs on spatial and temporal scales that are of experimental relevance. Furthermore, we discuss how the thicknesses of metal and of the substrate itself influence the maximum depth and liquid lifetime of the melted $\mathrm{SiO}_{2}$ layer. In particular, we find that there is a minimum thickness of $\mathrm{SiO}_{2}$ layer for which this layer melts and furthermore, the melting occurs only for metal films of thickness in a specified range. In the experiments, substrate melting is of practical importance since it may significantly modify the evolution of the deposited nanoscale metal films or other geometries on nanoscale.
\end{abstract}

Keywords: phase change, laser heating, nanoscale

Email addresses: francesc.font@math.mcmaster.ca (Francesc Font), shahriar.afkhami@njit.edu (Shahriar Afkhami), kondic@njit.edu (Lou Kondic) 


\section{Introduction}

Nanoparticle self-assembly, induced by dewetting of thin nanoscale metallic films, has recently been shown to be a promising process for synthesizing large-area nanoparticle ensembles, see Ref. 1 for a recent review. The process involves nanosecond pulsed-laser-induced melting of metallic films, filaments, and other geometries, followed by dewetting that may lead to formation of patterns characterized by a particular spatial order and size distribution $[2,3,4,5,6,7,8]$. Due to its relevance to a number of applications involving metal particles on nanoscale, the pulsed-laser dewetting has recently been studied in great detail $[9,10,11,12,13,14,15,16,17,18,19,20,21]$. While these studies lay out a clear picture of the basic physical mechanisms of the dewetting, understanding of relevant thermal phenomena is still limited, and there are only few studies discussing these effects $[4,10,22,12,23]$. In particular, to the authors' knowledge, not much attention has been paid to the thermal processes in the substrate itself, leading possibly to its melting. As an example motivating such a study, Fig. 1 shows an experimental image from Ref. 11 that suggests that high temperatures reached during laser irradiation may lead to partial melting of the substrate. This figure shows that after the initial laser pulse, the liquid metal filament recesses into the substrate, suggesting substrate melting. In such a scenario, the dewetting dynamics of the metal film can be strongly coupled to the substrate behavior, in particular since the dewetting of a metal film resting on a liquid substrate can be altered significantly. Therefore, there is a strong motivation to understand possible phase change of the substrate that may occur during experiments involving pulsed-laser irradiation of metal films and other geometries.

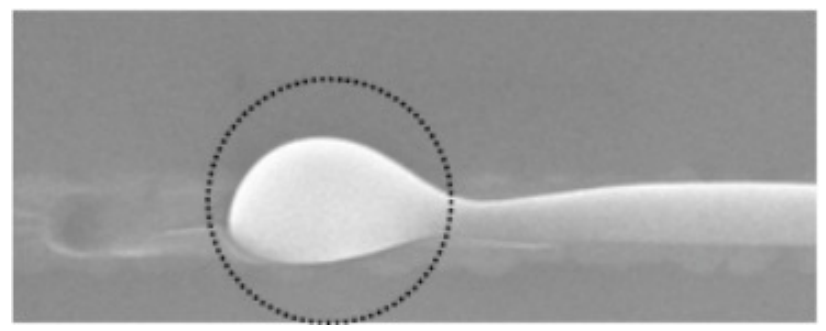

Figure 1: Experimental image from Ref. 11. After the initial laser pulse, the liquid metal filament (nickel) recesses slightly into the substrate ( $\mathrm{Si}$ with a $\mathrm{SiO}_{2}$ layer atop), suggesting substrate melting. 
In this work we present a model describing the heat transfer and phase change of the metal/substrate setup during laser irradiation. While for definitiveness we use the material parameters that correspond to a three layer setup consisting of copper $(\mathrm{Cu}) /$ silicon dioxide $\left(\mathrm{SiO}_{2}\right) / \operatorname{silicon}(\mathrm{Si})$ (see Fig. 2), we will vary appropriate nondimensional parameters in order to reach a more general understanding of the problem. Regarding $\mathrm{Cu} / \mathrm{SiO}_{2} / \mathrm{Si}$ setup, we note that while $\mathrm{SiO}_{2}$ may be native to the $\mathrm{Si}$ substrate, we concentrate here on a common setup such that an $\mathrm{SiO}_{2}$ layer of controlled thickness (typically 100 $\mathrm{nm})$ is applied to the Si substrate, see e.g. Ref. 24. The laser irradiation is considered to be applied via a Gaussian beam, and we study the effect of the laser parameters, including energy density and pulse duration, on possible melting of the $\mathrm{SiO}_{2}$ layer. For the parameters for which the $\mathrm{SiO}_{2}$ layer melts, we compute the depth of the melted $\mathrm{SiO}_{2}$ layer and the total time the layer remains in liquid phase. We find that maximum thickness of the melted region can be of the same order of magnitude as the thickness of the metal film. Finally, we study the effect of changing the metal and substrate thickness on the maximum depth and liquid lifetime of the melted $\mathrm{SiO}_{2}$ layer.

The rest of the paper is organized as follows. We begin in Sec. 2 by presenting the governing equations. In Sec. 3, we present the results, starting with the reference case (Sec. 3.1), followed by a discussion of the effects of key dimensionless variables, such as the ratio of the heat diffusion time to the laser pulse duration, and the ratio of energy per unit volume needed to melt the substrate to the energy per unit volume absorbed in the metal (Sec. 3.2). The role that the metal and substrate thicknesses play in melting process is discussed in Sec. 3.3. Section 4 summarizes the main conclusions.

\section{Mathematical model}

Figure 2 illustrates the considered setup. A metal thin film of the thickness $h_{m}$ is placed on top of a flat substrate of thickness $h_{c}$. The substrate consists of two layers - top layer of thickness $h_{s}\left(\mathrm{SiO}_{2}\right)$ that in turn lies on top of another layer of thickness $h_{c}-h_{s}(\mathrm{Si})$. The three-layer configuration, initially at room temperature, $T_{\text {room }}$, is irradiated by a Gaussian temporal laser pulse. Although our model is general and can be applied to any material, the $\mathrm{Cu} / \mathrm{SiO}_{2} / \mathrm{Si}$ experimental setup reported elsewhere, see e.g. Ref. 25, will be taken as a reference case. Thus, we will make references to $\mathrm{Cu}, \mathrm{SiO}_{2}$ and

Si layers throughout the paper to indicate metal, substrate 1 and substrate 2 layers, respectively. 
To keep the formulation of the problem tractable and as general as possible, we make a number of assumptions and approximations that are listed next. Future work should consider relaxing some of these, particularly if direct comparison to experiments is attempted. The assumptions are as follows: (i) the substrate is completely transparent to the laser radiation [26], so that the metal film absorbs the energy of the laser and transfers it to the substrate by conduction; (ii) we will consider static setup for both metal and the substrate, and therefore convection is not considered in modeling heat transfer (this assumption is justified a posteriori in Sec. 3.1); (iii) only heat transfer in the out-of-plane $(z)$ direction is considered, and (iv) the material properties are taken as temperature independent. For simplicity, for the metal film, we use the material parameters at ambient conditions; future works should consider the influence of the change of material properties with temperature on the results presented here; for reference, we note that, e.g., metal thermal conductivity changes about $15 \%$ between ambient and melting temperatures. Furthermore, we note that we focus on melting of the substrate itself, and do not discuss melting of the metal film that has been already considered in existing works $[4,12,17]$. These have also shown that the influence of phase change of metal on its temperature evolution is minor. Similar studies have also shown that laser energy typically used in experiments, ablation/evaporation of the metal film is negligible [27, 28]. For instance, for the reference case considered in this study the mass loss was estimated to be $<2 \%$ per laser pulse [28]. Therefore, thinning effects due to mass loss are not considered in the present manuscript. Finally, as we will discuss, the temperatures achieved at the $\mathrm{Cu} / \mathrm{SiO}_{2}$ interface will induce melting in the $\mathrm{SiO}_{2}$ layer but the melt front will not reach the Si layer below for the $\mathrm{SiO}_{2}$ thicknesses considered. Thus, we will focus on the configuration $\mathrm{Cu}$ /liquid- $\mathrm{SiO}_{2} /$ solid-SiO $2 /$ solid-Si, as depicted in Fig. 2.

To describe the heating and possible melting of the substrate, we consider heat conduction in each layer coupled by appropriate boundary and interface conditions. The laser-metal interaction is introduced by the means of a source term in the heat equation for the metal layer [29].

Under the above assumptions, the governing equations for the tempera- 


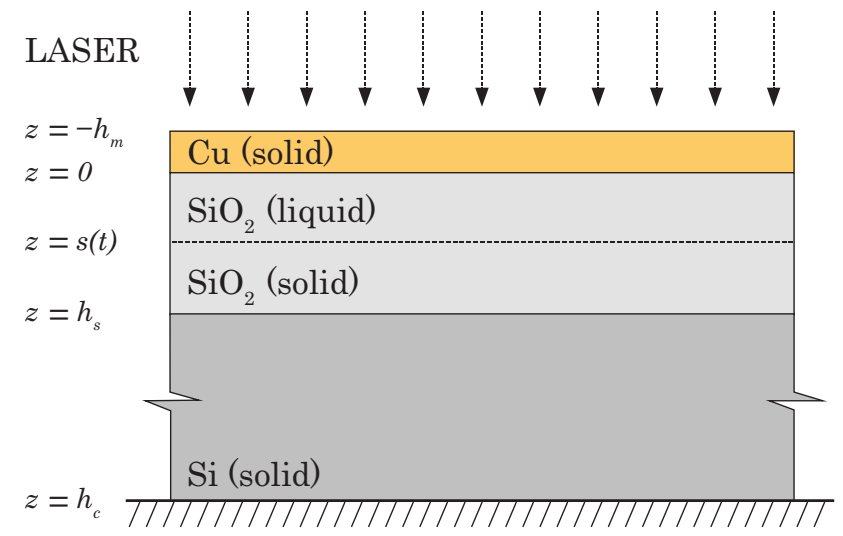

Figure 2: A schematic of the $\mathrm{Cu} / \mathrm{SiO}_{2} / \mathrm{Si}$ thin film structure, assuming that $\mathrm{SiO}_{2}$ layer is partially melted; the melting front is represented by the dashed line in the $\mathrm{SiO}_{2}$ layer (not to scale).

ture are as follows:

$$
\begin{aligned}
\rho_{m} c_{m} \frac{\partial T_{m}}{\partial t} & =k_{m} \frac{\partial^{2} T_{m}}{\partial z^{2}}+Q, \quad-h_{m}<z<0, \\
\rho_{l} c_{l} \frac{\partial T_{l}}{\partial t} & =k_{l} \frac{\partial^{2} T_{l}}{\partial z^{2}}, \quad 0<z<s(t), \\
\rho_{s} c_{s} \frac{\partial T_{s}}{\partial t} & =k_{s} \frac{\partial^{2} T_{s}}{\partial z^{2}}, \quad s(t)<z<h_{s}, \\
\rho_{c} c_{c} \frac{\partial T_{c}}{\partial t} & =k_{c} \frac{\partial^{2} T_{c}}{\partial z^{2}}, \quad h_{s}<z<h_{c} .
\end{aligned}
$$

where $T$ is the temperature, $k$ the thermal conductivity, $\rho$ the density and $c$ the specific heat. The subscripts $m, l, s, c$ indicate $\mathrm{Cu}$, liquid- $\mathrm{SiO}_{2}$, solid$\mathrm{SiO}_{2}$ and $\mathrm{Si}$, respectively. The phase change front is represented by $s(t)$.

The laser beam is applied in the $z$ direction. It is of the form [29]

$$
Q=E_{0}(1-R) g(x, y) f(z) q(t),
$$

where $E_{0}$ is the laser energy density and $R$ is the reflectivity of the $\mathrm{Cu}$ film. The attenuation within the metal is described by Beer's law, $f(z)=\alpha e^{-\alpha z}$, where $\alpha$ is the absorption coefficient [29]. The pulse is taken as Gaussian, and is specified by $q(t)=(1 / \sigma \sqrt{2 \pi}) \exp \left(-\left(t-t_{p}\right)^{2} / 2 \sigma^{2}\right)$. We choose the standard deviation, $\sigma=t_{p} / 2 \sqrt{2 \ln (2)}$, giving the full width at half maximum equal to $t_{p}$. 
The term $g(x, y)$ accounts for possible spatial variation of the pulse. For metals on nanoscale, the spatial extend of a laser spot is much larger than any other relevant lenthscale, motivating consideration of a uniform pulse, leading to $g(x, y)=1$. Therefore,

$$
Q=\frac{2 \sqrt{\ln (2)}(1-R) E_{0} \alpha}{t_{p} \sqrt{\pi}} e^{-4 \ln (2)\left(\frac{t}{t_{p}}-1\right)^{2}-\alpha\left(z+h_{m}\right)},
$$

where the reflectivity and absorption coefficient are given by

$$
R=\frac{(n-1)^{2}+\kappa^{2}}{(n+1)^{2}+\kappa^{2}}, \quad \alpha=\frac{4 \pi \kappa}{\lambda} .
$$

Here $n$ is the refractive index of the metal, $\kappa$ is the extinction coefficient, and $\lambda$ is the pulse wavelength. Note that the $z$ coordinate in Eq. (6) has been shifted so that the attenuation starts at the surface of the metal, at $z=-h_{m}$.

We assume perfect thermal contact between the three layers and neglect the interface thermal resistance effect, resulting in the following boundary conditions

$$
\begin{aligned}
& \left.k_{m} \frac{\partial T_{m}}{\partial z}\right|_{z=0}=\left.k_{l} \frac{\partial T_{l}}{\partial z}\right|_{z=0}, T_{m}(0, t)=T_{l}(0, t) \\
& \left.k_{s} \frac{\partial T_{s}}{\partial z}\right|_{z=h_{s}}=\left.k_{c} \frac{\partial T_{c}}{\partial z}\right|_{z=h_{s}}, T_{s}\left(h_{s}, t\right)=T_{c}\left(h_{s}, t\right) .
\end{aligned}
$$

Furthermore, we ignore heat loss at the metal surface and take the temperature at the bottom of the Si layer to be fixed at the room temperature. Thus,

$$
\left.\frac{\partial T_{m}}{\partial z}\right|_{z=-h_{m}}=0, \quad T_{c}\left(h_{c}, t\right)=T_{\text {room }} .
$$

Anticipating that $\mathrm{SiO}_{2}$ layer may melt, we also specify the following boundary conditions at the solid/liquid interface inside of the $\mathrm{SiO}_{2}$ layer

$$
\begin{gathered}
\rho_{s} L_{s} \frac{d s}{d t}=\left.k_{s} \frac{\partial T_{s}}{\partial z}\right|_{z=s(t)}-\left.k_{l} \frac{\partial T_{l}}{\partial z}\right|_{z=s(t)}, \\
T_{l}(s(t), t)=T_{s}(s(t), t)=T_{I}
\end{gathered}
$$


Table 1: Physical parameters for $\mathrm{Cu}, \mathrm{SiO}_{2}$ and $\mathrm{Si}$.

\begin{tabular}{|c||c|c|c|}
\hline \hline Material & $\begin{array}{c}c \\
(\mathrm{~J} / \mathrm{kg} \cdot \mathrm{K})\end{array}$ & $\begin{array}{c}\rho \\
\left(\mathrm{kg} / \mathrm{m}^{3}\right)\end{array}$ & $\begin{array}{c}k \\
(\mathrm{~W} / \mathrm{m} \cdot \mathrm{K})\end{array}$ \\
\hline \hline $\mathrm{Cu}$ & 390 & 8960 & 401 \\
$\mathrm{SiO}_{2}$ (liquid) & 420 & 2370 & 1.4 \\
$\mathrm{SiO}_{2}$ (solid) & 730 & 2200 & 1.4 \\
$\mathrm{Si}$ & 680 & 2300 & 150 \\
\hline \hline
\end{tabular}

where $T_{I}$ is the substrate melting temperature and $L_{s}$ the latent heat. Equation (11) results from the energy balance at the solid/liquid interface and is used to find the position of the phase change front. This equation requires the initial condition $s\left(t^{*}\right)=0$, where $t=t^{*}$ corresponds to the time when the melting starts, that is, when the $\mathrm{SiO}_{2}$ temperature at the $\mathrm{Cu} / \mathrm{SiO}_{2}$ interface reaches $T_{I}$. The pre-melting stage is modeled by removing the equation for the liquid and assuming $s(t)=0$. The numerical solution for this stage allows to pinpoint the time when melting starts and provides the profiles for $T_{m}\left(z, t^{*}\right), T_{s}\left(z, t^{*}\right)$ and $T_{c}\left(z, t^{*}\right)$ at the onset of melting. These profiles are then used as the initial conditions for Eqs. (1) - (4) and for Eq. (11).

Our reference case for the rest of the paper is motivated by the experimental setup discussed in Ref. 25. The laser properties are specified as follows: $\lambda=248 \mathrm{~nm}, t_{p}=18 \mathrm{~ns}$ and $E_{0}=1400 \mathrm{~J} / \mathrm{m}^{2}$, and layer thicknesses $h_{m}=16 \mathrm{~nm}, h_{s}=100 \mathrm{~nm}$, and $h_{c}=25 \mu \mathrm{m}$. The refractive index and extinction coefficient of $\mathrm{Cu}$ at wavelength $248 \mathrm{~nm}$ are $n=1.50$ and $\kappa=1.74$ [30]. The additional material properties are summarized in Table 1 . We note that the thermal conductivity of liquid $\mathrm{SiO}_{2}$ is very difficult to measure and previous studies show similar values for both solid and liquid conductivities [31]. For simplicity, we have taken them equal. The melting point and latent heat of $\mathrm{SiO}_{2}$ are given by $T_{I}=1883 \mathrm{~K}$ and $L_{s}=1.42 \cdot 10^{5} \mathrm{~J} / \mathrm{Kg}$ [32]. Also, we set the room temperature to $T_{\text {room }}=300 \mathrm{~K}$.

\subsection{Nondimensionalization}

By introducing the nondimensional variables

$$
\hat{T}=\frac{T-T_{\text {room }}}{\Delta T}, \quad \hat{t}=\frac{t}{\tau}, \quad \hat{z}=\frac{z}{L},
$$


and defining the scales

$$
\begin{array}{r}
L=h_{s}, \quad \tau=\frac{h_{s}^{2} \rho_{s} c_{s}}{k_{s}}, \\
\Delta T=\frac{2 \sqrt{\ln (2)}(1-R) E_{0} \alpha h_{s}^{2}}{t_{p} \sqrt{\pi} k_{m}},
\end{array}
$$

the governing equations, Eqs. (1) - (4) become, dropping the "^" notation,

$$
\begin{aligned}
a_{1} \frac{\partial T_{m}}{\partial t} & =\frac{\partial^{2} T_{m}}{\partial z^{2}}+q, \quad-l_{1}<z<0, \\
a_{2} \frac{\partial T_{l}}{\partial t} & =\frac{\partial^{2} T_{l}}{\partial z^{2}}, \quad 0<z<s(t), \\
\frac{\partial T_{s}}{\partial t} & =\frac{\partial^{2} T_{s}}{\partial z^{2}}, \quad s(t)<z<1, \\
a_{3} \frac{\partial T_{c}}{\partial t} & =\frac{\partial^{2} T_{c}}{\partial z^{2}}, \quad 1<z<l_{2} .
\end{aligned}
$$

where $q=e^{-4 \ln (2)(r t-1)^{2}} e^{-\alpha h_{s}\left(z+l_{1}\right)}, l_{1}=h_{m} / h_{s}, l_{2}=h_{c} / h_{s}, a_{1}=k_{l} \rho_{m} c_{m} / k_{m} \rho_{l} c_{l}$, $a_{2}=k_{s} \rho_{l} c_{l} / k_{l} \rho_{s} c_{s}$, and $a_{3}=k_{s} \rho_{c} c_{c} / k_{c} \rho_{s} c_{s}$. Upon nondimensionalization, Eqs. (8) - (12) read as

$$
\begin{aligned}
& \left.\frac{\partial T_{m}}{\partial z}\right|_{z=0}=\left.k_{1} \frac{\partial T_{l}}{\partial z}\right|_{z=0}, \quad T_{m}(0, t)=T_{l}(0, t), \\
& \left.k_{3} \frac{\partial T_{s}}{\partial z}\right|_{z=1}=\left.\frac{\partial T_{c}}{\partial z}\right|_{z=1}, \quad T_{s}(1, t)=T_{c}(1, t), \\
& \left.\frac{\partial T_{m}}{\partial z}\right|_{z=-l_{1}}=0, \quad T_{c}\left(l_{2}, t\right)=0, \\
& \frac{p}{r} \frac{d s}{d t}=\left.\frac{\partial T_{s}}{\partial z}\right|_{z=s(t)}-\left.k_{2} \frac{\partial T_{l}}{\partial z}\right|_{z=s(t)}, \\
& T_{l}(s(t), t)=T_{s}(s(t), t)=\tilde{T}_{I},
\end{aligned}
$$

where $k_{1}=k_{l} / k_{m}, k_{2}=k_{l} / k_{s}, k_{3}=k_{s} / k_{c}, \tilde{T}_{I}=\left(T_{I}-T_{\text {room }}\right) / \Delta T$ and

$$
r=\frac{\tau}{t_{p}}, \quad p=\frac{\sqrt{\pi} L_{s} \rho_{s} k_{m}}{2 \sqrt{\ln (2)}(1-R) E_{0} \alpha k_{s}} .
$$

Here, $r$ represents the ratio of diffusion time to the laser pulse duration, and $p$ is a parameter proportional to the ratio of energy needed to melt the 
substrate, $\rho_{s} L_{s}$, to the energy absorbed in the metal, $(1-R) E_{0} \alpha$. In the next section we will discuss the relative effect of changing the laser energy and pulse duration on the maximum depth and liquid lifetime of the melted $\mathrm{SiO}_{2}$ layer by independently varying $p$ and $r$.

In the heat transfer literature, the factor $p / r$ is usually given in terms of the Stefan number, $\beta=L_{s} / c_{s} \Delta T$. This formulation is useful, for instance, when modeling situations where the melting of a single layer material is triggered by applying a constant temperature $T_{H}$ above the melting temperature $T_{I}$ on one end of the material. Then, the scale $\Delta T=T_{H}-T_{I}$ describes the maximum temperature change in the material and $c_{s} \Delta T$ represents the sensible heat (per unit mass). By setting $p / r=\beta$ in Eq. (23) we realize that $d s / d t \propto 1 / \beta$, where $d s / d t$ represents the speed of the melting front. If the sensible heat (controlled by $T_{H}$ ) is much larger than the latent heat, then $\beta \ll 1$ and the melting will proceed fast. On the contrary, if $\beta \gg 1$ the melting will proceed slowly. In other words, the value of the Stefan number gives a measure of the speed of the melting process [33].

While the classical formulation described above is often very useful, in the present case discussing the problem in terms of the Stefan number would not allow us to fully decouple the effects caused by changing the laser pulse duration from those changing the laser energy. For this reason, we resort to the formulation based on $(p, r)$ as the control parameters.

\section{Results and discussion}

When a laser pulse is applied, the temperature of the metal film increases. If the temperature of the metal is raised above the substrate melting point, $T_{I}$, the substrate starts melting. The melting continues through the laser pulse duration, until the maximum of the pulse has passed and the temperature starts to decrease. The substrate starts to solidify and once the temperature at the metal/substrate interface is below $T_{I}$, re-solidification is completed. In this section, we discuss the details of this process. We will see that the results are not at all obvious, and include non trivial dependence of the depth of the melted region, as well as of the total liquid lifetime, on the parameters entering the problem.

The numerical solutions of Eqs. (16), (18) and (19) for $s(t)=0$ are obtained by implementing finite difference discretization (with implicit time integration) allowing to pinpoint the time when melting starts, $t^{*}$, and providing the profiles for $T_{m}, T_{s}$ and $T_{c}$ at the onset of melting, that are used 
as initial conditions to solve the melting problem. When melting begins, the model requires solving Eqs. (17) and (18) over the time-dependent domains $[0, s(t)]$ and $[s(t), 1]$, respectively. In doing so, we first fix the boundaries of $T_{l}$ and $T_{s}$ by implementing the following change of variables: $\xi=z / s$ and $\eta=(z-s) /(1-s)$. Then, we apply a semi-implicit finite difference scheme that discretizes implicitly $T_{l}$ and $T_{s}$ and explicitly $s(t)[34,35]$. The melting process does not affect the domain boundaries of $T_{m}$ and $T_{c}$, so Eqs. (16) and (19) are solved by standard backward Euler finite differences.

The numerical solution of the model allows to track the position of the melting front. In what follows, we focus on the maximum depth of the melted region, $s_{\text {max }}$, and the liquid lifetime, $t_{L}$, of the $\mathrm{SiO}_{2}$ layer. First, we discuss the results obtained for the reference case that we have defined in the preceding section. Then, we explore the effects of the laser fluence and the pulse duration. Finally, we study the role that the metal and substrate thicknesses play in determining the maximum depth and liquid lifetime of the molten region.

\subsection{Phase change: the reference case}

Figure 3 shows the temperature distribution across the four layers $(\mathrm{Cu}$, molten $\mathrm{SiO}_{2}$, solid $\mathrm{SiO}_{2}$, and $\mathrm{Si}$ ) at various stages of the phase change. The position of the phase change front, $s(t)$, is indicated by the dashed vertical line. Figure 3 shows that the large temperature drop occurs in the $\mathrm{SiO}_{2}$ layer, $T_{s}(1, t)-T_{l}(0, t) \approx 15$, while the temperatures in the $\mathrm{Cu}$ and $\mathrm{Si}$ show virtually flat profiles, $T_{m}(0, t)-T_{m}\left(-l_{1}, t\right) \approx 0.01$ and $T_{c}\left(l_{2}, t\right)-T_{c}(1, t) \approx 1$. To put these values in perspective, we note that for the reference case, the relevant scales are given by $\tau=11.47 \mathrm{~ns}, \Delta T=103.96 \mathrm{~K}$, and $L=100 \mathrm{~nm}$. In addition, for the reference case, we have that $r=0.64$ and $p=1.20$. We note that $\tau$ and $t_{p}$ are of the same order of magnitude, showing that the evolution of the melting front occurs on the time scale comparable to the laser pulse duration.

The fact that temperature varies only slightly through the metal film is as expected due to high conductivity of the $\mathrm{Cu}$ layer and its small thickness. Also, during the laser pulse, the temperature within both the solid and liquid regions of the $\mathrm{SiO}_{2}$ appears to be almost linear in $z$. This is due to the the fact that the time scale on which the temperature reaches steady state value is much faster than the time scale describing motion of the phase change front. Finally, small variation of the temperature in the Si layer is the result of the low thermal conductivity of $\mathrm{SiO}_{2}$, leading to rather minor amount of heat 

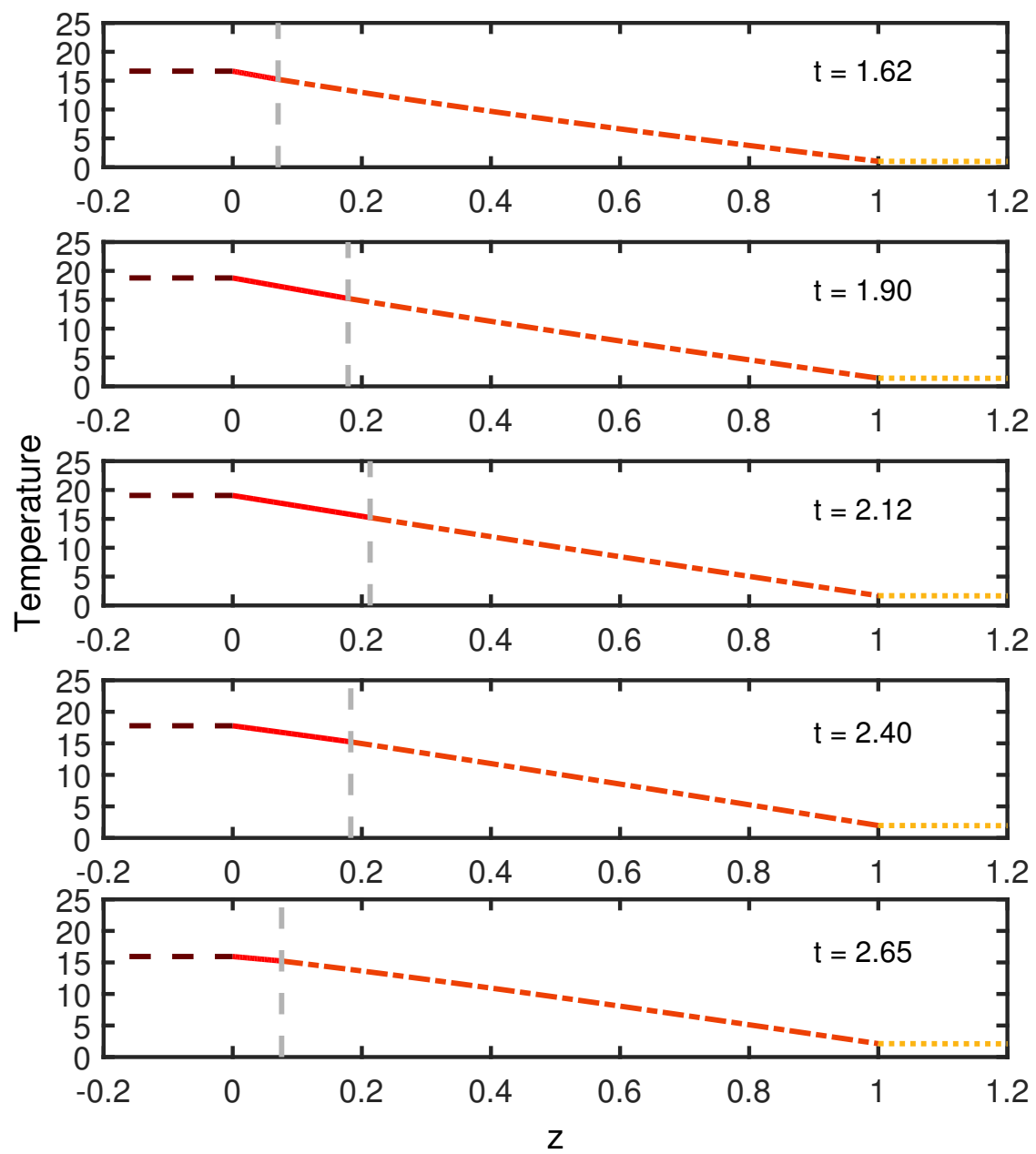

Figure 3: Temperature in the four regions $\left(\mathrm{Cu}\right.$, molten $\mathrm{SiO}_{2}$, solid $\mathrm{SiO}_{2}$, and $\left.\mathrm{Si}\right)$ at various stages of melting for the reference case. The gray vertical line indicates the position of the melting front. The temperature in the Si region does not provide valuable information for large $z$ and has been truncated at $z=1.2$. In this and all the following figures the parameters corresponding to the reference case are used, if not specified differently. The noted values of $t$ measure the time since the laser is switched on. For the reference case, melting begins at $t=t^{*}=1.5$. 
conducted through the $\mathrm{SiO}_{2} / \mathrm{Si}$ interface. We note in passing that due to the diffusion characteristic time scale for the Si layer (given by $\left(h_{c}-h_{s}\right)^{2} c_{c} \rho_{c} / k_{c} \approx$ $6.64 \mu \mathrm{s})$ being much larger than the laser time scale $\left(t_{p}=18 \mathrm{~ns}\right)$, a boundary layer near $z=1$ appears. Since such a boundary layer is not central to the problem on which we focus, we do not show it in Fig. 3, nor we discuss it in more detail in the present paper.

Figure 4(a) shows the evolution of the temperature at $z=0$, with the horizontal line indicating the melting temperature of the substrate, $T_{I}$. The dashed line corresponds to the numerical solution of the full model and the solid line shows the results obtained if the phase change is ignored (by explicitly setting $s(t)=0$ ). During the phase change stage, a part of the energy delivered by the laser is used to transform solid into liquid rather than for increasing the temperature. Therefore, the temperature predicted by the model when setting $s(t)=0$ (solid line), i.e., not taking the $\mathrm{SiO}_{2}$ phase change into account, is higher than the temperature predicted by the full model (dashed line). The fact that the temperatures are similar suggests only minor effect of melting on the energy flow in the system, similarly as noted for melting of the metal itself [4].

Figure 4(b) shows the evolution of the phase change front for the reference case. The peak in $s(t)$ corresponds to the maximum thickness of the molten region, $s_{\max } \approx 0.22$; with our choice of length scale, this value corresponds to $22 \mathrm{~nm}$. The liquid lifetime is defined as $t_{L}=t_{\text {end }}-t^{*}$, where $t_{\text {end }}$ is the time when re-solidification is over. For the reference case, $t^{*}=1.5$ and $t_{\text {end }}=2.76$ leading to the liquid lifetime of $t_{L}=1.26$, corresponding to 14.49 ns. Therefore, a significant part of the substrate is melted, during the time interval that is comparable to the pulse duration, $t_{p}$.

We note that the time scales considered in the present work are short compared to the ones on which one could expect that thermocapillary stresses play a role, possibly modifying film thickness. To see this, one could consider linear stability analysis (LSA) of a flat film, and discuss the time scale on which a small perturbation grows; such a time scale, $\tau_{L S A}$, is usually approximated by the inverse of the maximum growth rate computed by LSA, $\sigma_{m}$. Considering, for example, Ref. 23, one finds that (in dimensional form) $\tau_{L S A}=\left(3 \mu h_{m} / \gamma_{0}\right) / \sigma_{m}$, with $\sigma_{m}=G^{2}\left(h_{m} / 4\right)\left[3 \gamma_{T} /\left(2 \gamma_{0}\right)\right]^{2}$ where $G$ measuring $d T / d h$ is the quantity of $\mathrm{O}(1-10)$ [23]. The parameters $\mu$, $\gamma_{0}$ and $\gamma_{T}$ correspond to the viscosity, surface tension and surface tension dependence on temperature, respectively. Plugging in the numerical values $\gamma_{T}=-2.3 \cdot 10^{-4} \mathrm{~J} \mathrm{~m}^{-2}, \gamma_{0}=1.3 \mathrm{~J} \mathrm{~m}^{-2}$, and $\mu=4.3 \cdot 10^{-3} \mathrm{~m}^{2} \mathrm{~s}^{-1}$ (from Table 


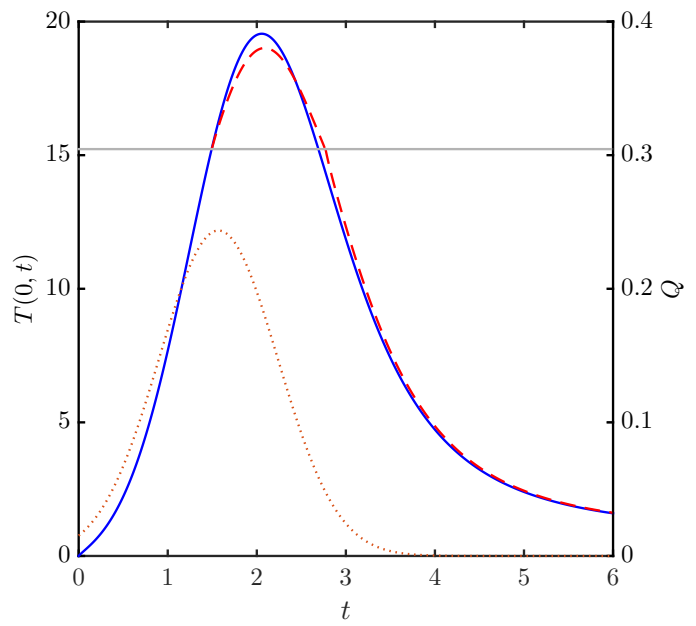

(a)

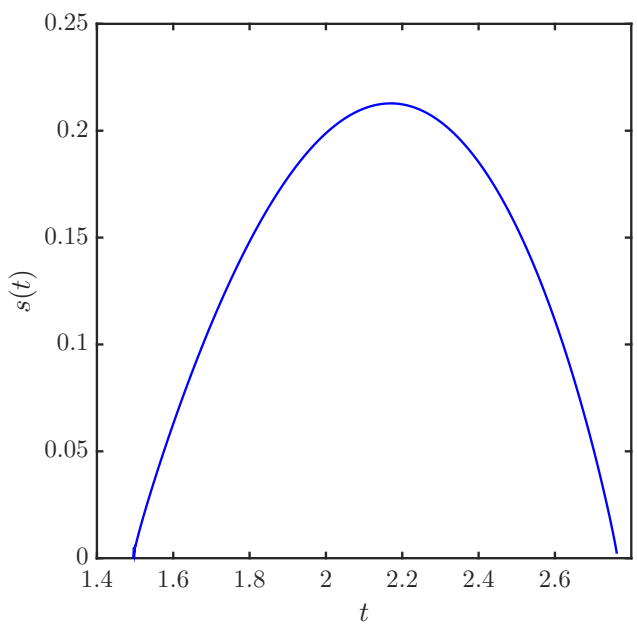

(b)

Figure 4: (a) Evolution of the temperature at the $\mathrm{Cu} / \mathrm{SiO}_{2}$ interface $(z=0)$. Blue solid line shows the temperature obtained from the model when phase change is ignored, and the red dashed line is the solution of the full model. The dotted line represents the source term (right $y$-axis) in Eq. (16) and the horizontal line depicts the melting temperature. (b) Evolution of the phase change front, $s(t)$. 
I in Ref. 23) gives the time scale of $10^{-7}-10^{-6}$ sec, which is at least an order of magnitude longer than the time scales considered here. Therefore, it is reasonable to assume that film thickness is constant on the time scales of relevance in the present work.

Using the time scale $\tau_{L S A}$, we can also estimate the reference speed $u=$ $L / \tau_{L S A}$ characterizing convective flow in the metal layer; here we take $L \approx$ $100 \mathrm{~nm}$ as a typical length scale relevant to the fluid flow (this $L$ is of the same order of magnitude as the most unstable wavelength resulting from the LSA discussed above for a $10 \mathrm{~nm}$ thick film). By defining the Peclet number as $P e=u h_{m} / \alpha_{m}$ where $\alpha_{m}=k_{m} / c_{m} \rho_{m}$ is the thermal diffusivity of the metal layer, we obtain $P e$ is $\mathrm{O}\left(10^{-5}-10^{-6}\right)$, thereby confirming that convective effects are indeed negligible for the setup considered in the present work.

\subsection{The influence of laser pulse properties}

The nondimensionalization of the model has produced the dimensionless parameters $r$ and $p$, which are related to the pulse duration and the fluence of the laser source. In particular, $r \propto 1 / t_{p}$ and $p \propto 1 / E_{0}$. Indeed, different combinations of values for $r$ and $p$ will lead to different liquid lifetimes and thicknesses of the molten region.

Figure 5 provides $r$ - $p$ phase diagrams with superimposed $t_{L}$ and $s_{\max }$ contours; it is useful to consider this figure jointly with Fig. 6 that shows a cross-section of Fig. 5 for a fixed value of $p$, as well as plots of some additional quantities discussed below. Recall that the reference case corresponds to $(r, p)=(0.64,1.20)$. In Fig. 5, the central red diagonal stripe shows the range that corresponds to partial melting of the $\mathrm{SiO}_{2}$. The orange area in the upper left corner corresponds to the $p-r$ combinations for which melting of $\mathrm{SiO}_{2}$ does not occur. The brown area on the lower right corner shows the part of the space where the temperatures are higher than the boiling point of $\mathrm{Cu}$. Since our model does not apply to such high temperatures, we will not discuss this part of the parameter space any further.

To understand the behavior of $t_{L}$ and $s_{\text {smax }}$ contours in Fig. 5, we first write Eq. (6) in terms of the temperature scale, $\Delta T$, as

$$
Q=\frac{k_{m}}{h_{s}^{2}} \Delta T e^{-4 \ln (2)(r t-1)^{2}-\alpha h_{s}\left(z+l_{1}\right)} .
$$

Then, by combining Eqs. (15) and (25), we observe that the temperature scale can be expressed as $\Delta T=L_{s} r /\left(c_{s} p\right)$, leading to $Q \propto(r / p) \exp \left(-4 \ln (2)(r t-1)^{2}\right)$. 


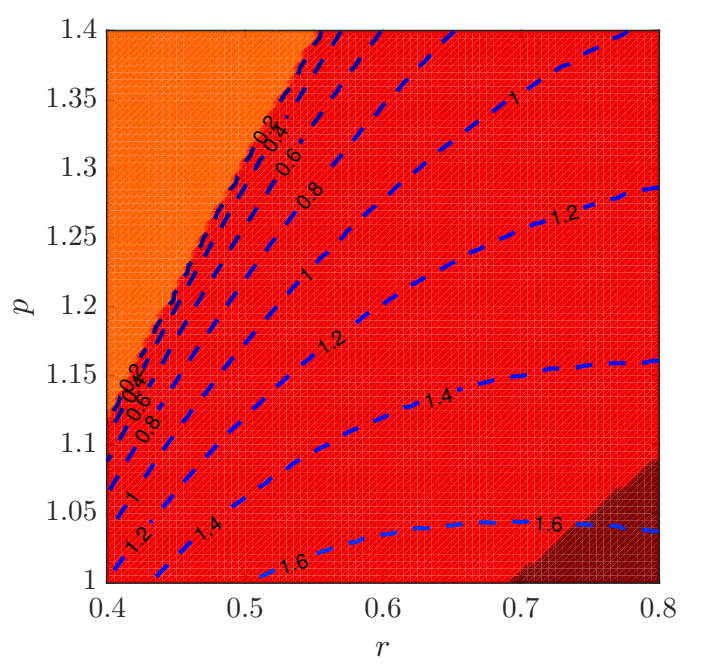

(a)

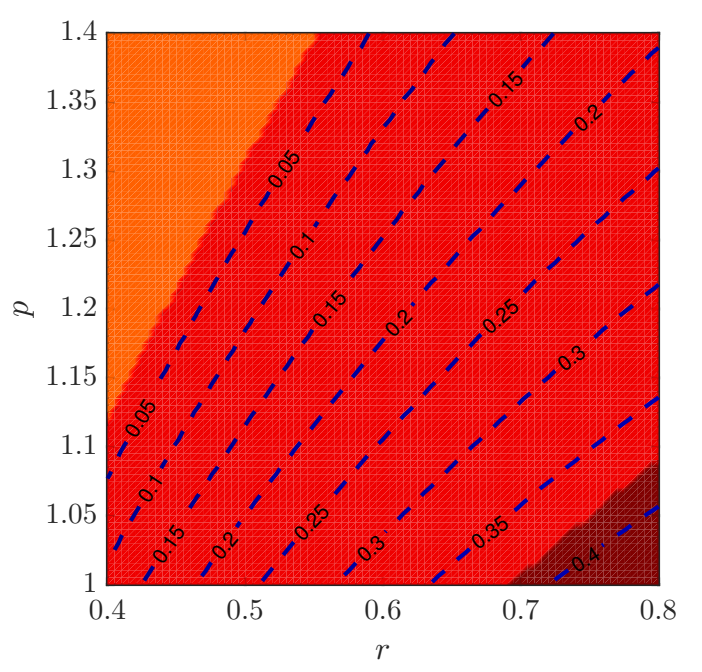

(b)

Figure 5: Phase diagram in $p-r$ space. Orange area on the upper left corner represents the region where the $\mathrm{SiO}_{2}$ substrate does not reach the melting temperature and remains solid. The central red area represents the region where $\mathrm{SiO}_{2}$ melts. The darker area on the lower right corner represents the region where the temperature of the melted $\mathrm{SiO}_{2}$ goes above the boiling temperature of $\mathrm{Cu}$ (where the present model is not expected to hold). The contour lines represent combinations of $p-r$ leading to fixed liquid life time, $t_{L}$, (a) and fixed thickness, $s_{\max }$, (b) of melted $\mathrm{SiO}_{2}$. 
Note also that, using Eq. (6), we can write $Q \propto\left(E_{0} / t_{p}\right) \exp \left(-4 \ln (2)\left(t / t_{p}-1\right)^{2}\right)$. Based on these expressions for $Q$, we realize that changes in $p$ only affect the amplitude of the Gaussian pulse, while the changes in $r$ affect both the amplitude and the pulse duration.

The observations given in the previous paragraph allow us to better understand the general features of the results shown in Fig. 5. We see that increasing $r$ while keeping $p$ fixed, or decreasing $p$ with fixed $r$, produces larger $s_{\max }$; see also Fig. 6a. This is due to the fact that increasing $r$ or decreasing $p$ results in larger amplitudes $r / p$ of the Gaussian pulse, so more energy is delivered to the system. However, by comparing Figs. 5(a) and (b), it can be seen that $t_{L}$ increases only for small values of $r$ (keeping $p$ fixed), while for larger $r$ 's, the liquid lifetime does not increase. The reason for this can be seen in Fig. 6(d) that shows the variation of the Gaussian pulse with $r$ for fixed $p$. As $r$ increases, the pulse amplitude increases and the duration of the pulse decreases, leading to a larger amount of energy delivered faster. Thus $s_{\max }$ becomes larger, but the liquid lifetime does not necessarily increase since the pulse is shorter.

It is also relevant to discuss how the phase change process affects the energy flux through the $\mathrm{SiO}_{2} / \mathrm{Si}$ interface. Figure 6(b) shows the energy flux $\psi(z=1, t)=\partial T_{c} / \partial z$ for the same values of $r$ as in Fig. 6(a). The results show that the energy flux is higher when $s_{\max }$ is smaller, as less energy has been spent in phase change. To simplify the comparison, the curves in Figs. 6(a) - (b) have been aligned with respect to $t^{*}$, the time when melting starts. We note that for large $r$ (more energy, shorter pulse) the melting process starts earlier than for small $r$ (less energy, longer pulse); see also Fig. 6(c).

\subsection{The dependence of phase change process on metal and $\mathrm{SiO}_{2}$ thickness}

In the experiments involving metal films on Si substrates, the thicknesses of the metal film and the deposited $\mathrm{SiO}_{2}$ layer could be easily controlled and varied. Therefore, we next discuss the dependence of $t_{L}$ and $s_{\max }$ on these quantities. Figure 7 shows the changes in $t_{L}$ and $s_{\max }$ as we vary the metal thickness from $l_{1}=0.09$ to $l_{1}=0.82$ (or equivalently from $9 \mathrm{~nm}$ to $82 \mathrm{~nm}$ ) in steps of $\Delta l_{1}=0.01$. For smaller values of $l_{1}$, we see that increasing $l_{1}$ results in an increase in $t_{L}$ and $s_{\max }$. However, if we keep increasing $l_{1}$, both $t_{L}$ and $s_{\max }$ reach a maximum and then start decreasing. The explanation for this non-monotonous dependence is that the amount of energy that the metal can absorb is limited by the Beer's attenuation factor, $e^{-\alpha h_{s}\left(z+l_{1}\right)}$, in the source term. For smaller $l_{1}$, an increase in the 


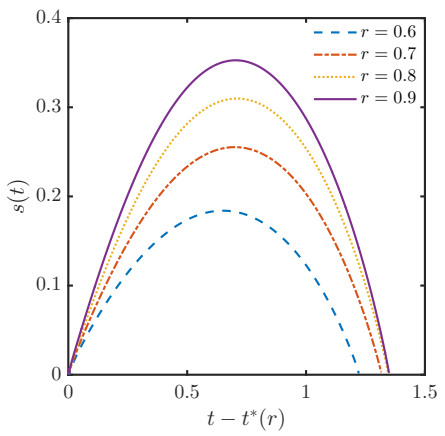

(a)

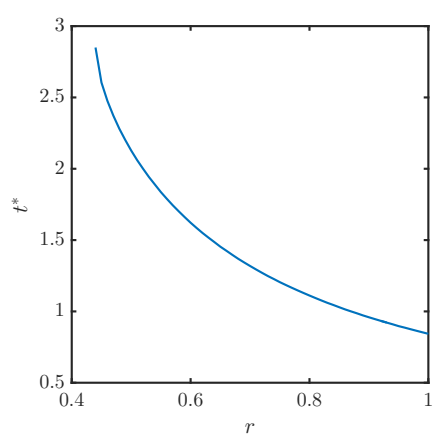

(c)

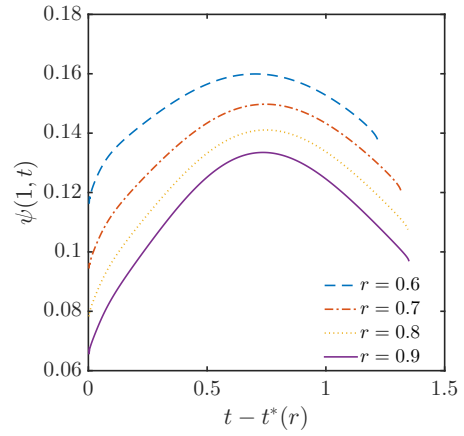

(b)

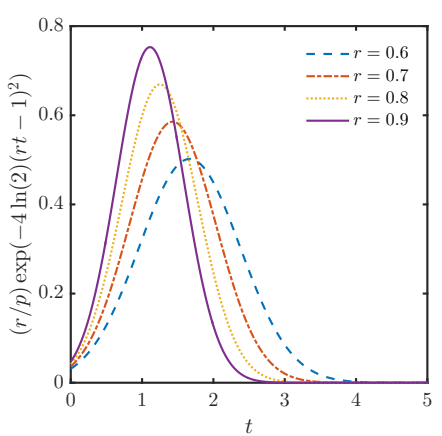

(d)

Figure 6: (a) Position of the phase change front for several values of $r$. (b) The heat flux through the $\mathrm{SiO}_{2} / \mathrm{Si}$ interface $(z=1)$. The melting starts at $t^{*}(r)$, and we have conveniently aligned all the curves in (a) and (b) to start from $t=t^{*}$. In (c), we show how $t^{*}$ changes as a function of $r$. (d) Source term evolution at $z=0 . p=1.2$ is used throughout. 


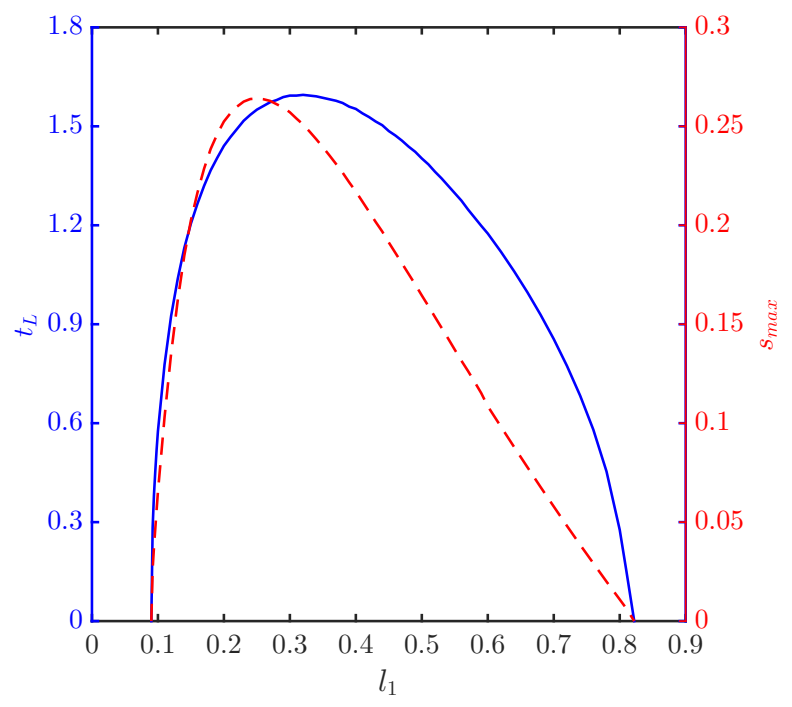

Figure 7: The dependence of $t_{L}$ and $s_{\max }$ on the metal thickness $l_{1}$. The solid line represents the liquid lifetime (left $y$-axis) and the dashed line the maximum depth (right $y$-axis) of molten $\mathrm{SiO}_{2}$ as a function of metal thickness, $l_{1}$.

metal thickness leads to an increase of energy absorbed, which is then used to heat up the metal and the substrate and consequently leads to an increase of $t_{L}$ and $s_{\max }$. For sufficiently large $l_{1}$ there will be no further significant increase in the energy absorbed, but the amount of metal that needs to be heated is larger, and therefore the temperature drops, and the liquid lifetime and the depth of the molten region decrease. The consequence of this nonmonotonous dependence is that melting of the substrate occurs only for a range of metal thicknesses - Fig. 7 shows that the minimum metal thickness for which substrate melting occurs is $\approx 0.09 \sim 9 \mathrm{~nm}$ and the maximum $\approx 0.82 \sim 82 \mathrm{~nm}$; clearly, as the properties of the laser source, or the $\mathrm{SiO}_{2}$ thickness (discussed next) are modified, this range changes as well.

Note that the turnaround points in $t_{L}$ and $s_{\max }$ are achieved at different values of $l_{1}$. In particular, $t_{L}$ shows maximum at $l_{1} \approx 0.32$ and $s_{\max }$ at $l_{1} \approx 0.25$, corresponding approximately to $32 \mathrm{~nm}$ and $25 \mathrm{~nm}$, respectively. Figure 8(a) shows the reason for this difference. We see that the temperature of the $\mathrm{Cu} / \mathrm{SiO}_{2}$ interface for $l_{1}=0.25$, at which the maximum of $s_{\max }$ occurs, reaches the maximum value. On the other hand, for $l_{1}=0.32$, at which the maximum of $t_{L}$ occurs, we find that the interface temperature is higher than 


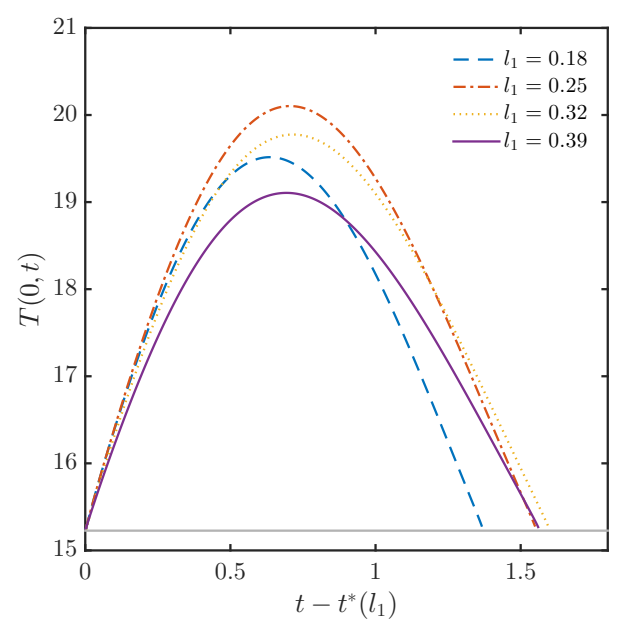

(a)

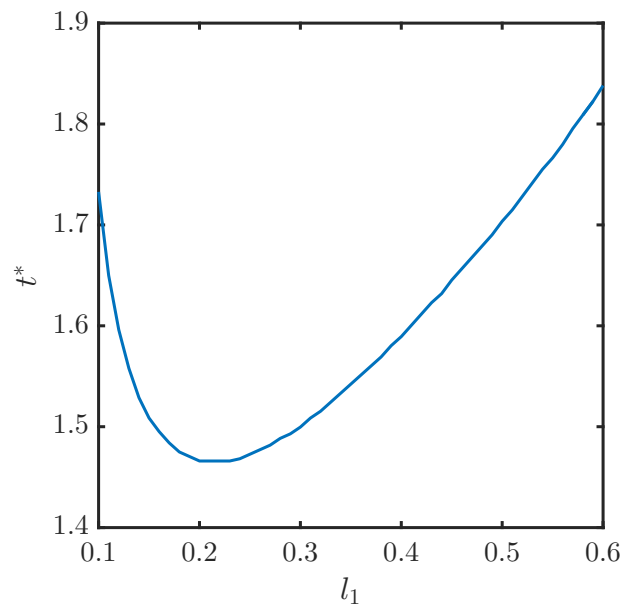

(b)

Figure 8: (a) Temperature evolution of the $\mathrm{Cu} / \mathrm{SiO}_{2}$ interface during phase change for several values of metal thickness, $l_{1}$. (b) The time at which melting starts, $t^{*}$. 


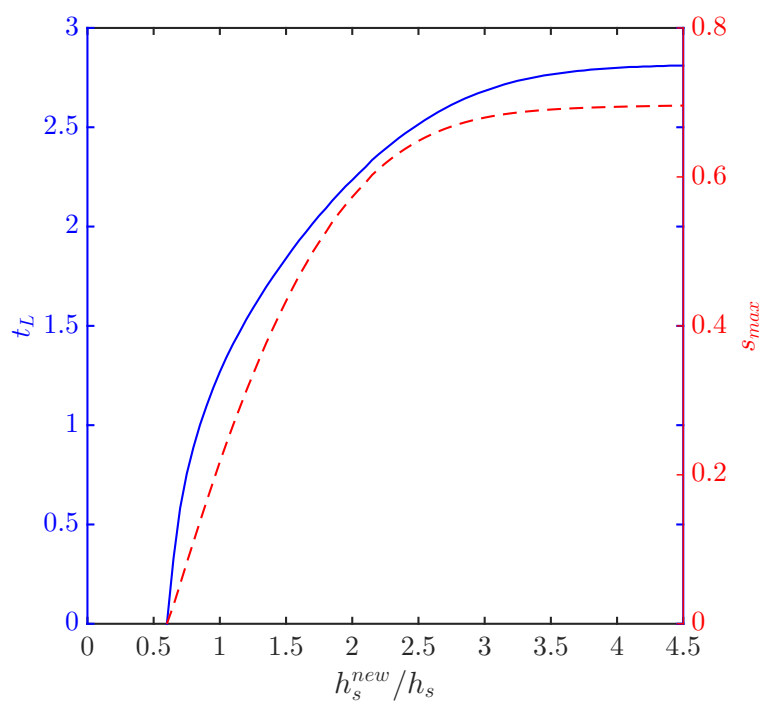

Figure 9: The dependence of $t_{L}$ and $s_{\max }$ on the modified $\mathrm{SiO}_{2}$ thickness with respect to the reference $\mathrm{SiO}_{2}$ thickness, $h_{s}^{\text {new }} / h_{s}$. The solid line represents the liquid lifetime (left $y$-axis) and the dashed line the maximum depth (right $y$-axis) of molten $\mathrm{SiO}_{2}$ as a function of the substrate thickness, $h_{s}^{\text {new }} / h_{s}$.

$T_{I}$ for the (slightly) longer period. We note that the curves in Fig. 8(a) have been aligned with respect to $t^{*}$, the time when melting starts, see Fig. 8(b); the results shown in this figure could be rationalized similarly as the results for $t_{L}$ and $s_{\max }$ in Fig. 7 .

Next we focus on the influence of the $\mathrm{SiO}_{2}$ thickness. Figure 9 shows how $t_{L}$ and $s_{\max }$ vary as the thickness of the $\mathrm{SiO}_{2}$ is modified with respect to the reference $\mathrm{SiO}_{2}$ thickness; we call the modified $\mathrm{SiO}_{2}$ thickness $h_{s}^{\text {new }}$. We observe that for large values of $h_{s}^{\text {new }} / h_{s}, t_{L}$ and $s_{\max }$ saturate. This is as expected, since for a sufficiently large $h_{s}^{\text {new }}$ the $\mathrm{SiO}_{2}$ layer is thick enough to be considered as of effectively infinite thickness, and due to small heat conductivity of $\mathrm{SiO}_{2}$, the influence of the boundary condition at the bottom of the substrate disappears.

Figure 9 also shows that $t_{L}$ and $s_{\max }$ go to zero when $h_{s}^{\text {new }} / h_{s} \approx 0.63$, meaning that melting of the $\mathrm{SiO}_{2}$ layer is suppressed if its thickness is smaller than $h_{s}^{\text {new }} \approx 0.63 h_{s}=63 \mathrm{~nm}$. Therefore, our results indicate that there exists a minimum thickness of the $\mathrm{SiO}_{2}$ layer at which the melting of the substrate can be completely avoided. Clearly, the exact critical value of the $\mathrm{SiO}_{2}$ 
thickness depends on the metal film thickness as well as on the laser source properties.

To better understand the influence of $\mathrm{SiO}_{2}$ thickness on melting, Fig. 10 shows the evolution of the temperature during phase change at both ends of the $\mathrm{SiO}_{2}$ layer $(z=0$ and $z=1)$ for decreasing values of $h_{s}^{\text {new }} / h_{s}$. For small values of $h_{s}^{\text {new }} / h_{s}$, the heat is easily transported through the $\mathrm{SiO}_{2}$ to the $\mathrm{Si}$ substrate, which translates into lower temperatures of the $\mathrm{Cu} / \mathrm{SiO}_{2}$ interface (part (a)) and higher temperatures at the $\mathrm{SiO}_{2} / \mathrm{Si}$ interface (part (b)). When $h_{s}^{\text {new }} / h_{s}<0.63$ the temperature at the $\mathrm{Cu} / \mathrm{SiO}_{2}$ interface does not reach the phase change temperature $T_{I}$ and melting of the substrate does not occur.

\section{Conclusions}

Metal films of nanoscale thickness evolving on top of Si substrates covered by $\mathrm{SiO}_{2}$ are becoming increasingly important in a number of applications, and have been considered commonly in laboratory experiments. In this paper we have presented the results that suggest that the $\mathrm{SiO}_{2}$ part of the substrate may go through phase change process under the conditions commonly used in the experiments and applications involving laser irradiated metal films on nanoscale. We focus on understanding the details of the melting process, and how the extend and duration of melting depend on the material parameters, properties of the laser source, and the thicknesses of the metal film and of the $\mathrm{SiO}_{2}$ layer. Particularly interesting finding is that, everything else kept fixed, there is a specified range of metal thickness, and a minimum value of the $\mathrm{SiO}_{2}$ thickness, for which melting occurs.

Melting of the substrate in the experiments involving dynamics of metal films may have significant consequence on the metal film dynamics (due to thermocapillary stresses, or some other driving force), since the metal film may be evolving on liquid, instead of solid substrate. Clearly, the evolution itself will lead to more complex setups since the thickness of a metal film becomes spatially dependent, influencing the heat flow and melting process. The present work sets a stage for considering such more complicated problems involving both heat and fluid flow. 


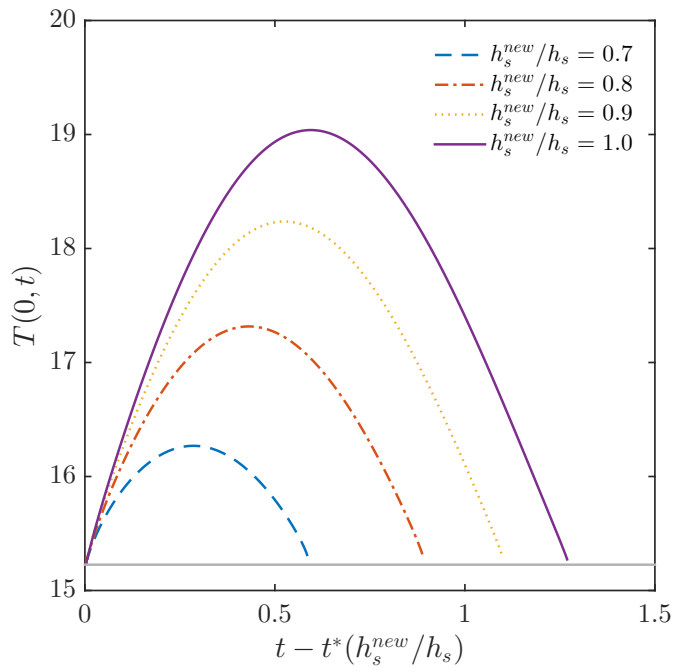

(a)

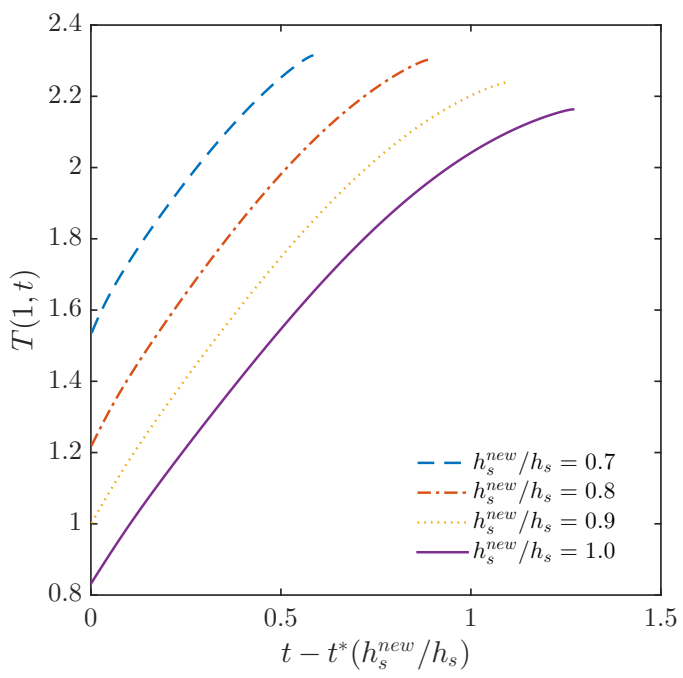

(b)

Figure 10: Temperature evolution of the $\mathrm{Cu} / \mathrm{SiO}_{2}$ (a) and the $\mathrm{SiO}_{2} / \mathrm{Si}$ (b) interface as the $\mathrm{SiO}_{2}$ thickness is modified, with respect to the reference $\mathrm{SiO}_{2}$ thickness, $h_{s}^{\text {new }} / h_{s}$. The horizontal line in (a) represent the melting temperature, $T_{I}$. 


\section{Acknowledgement}

F. F. acknowledges a mobility grant (Borsa Ferran Sunyer $i$ Balaguer 2013) from the Fundació Ferran Sunyer i Balaguer of the Institute of Catalan Studies. This work is partially supported by the NSF grants No. CBET1604351 (L. K., S. A.) and DMS-1320037 (S. A.).

\section{References}

[1] C. V. Thompson. Solid-state dewetting of thin films. Annual Review of Materials Research, 42(1):399-434, 2012.

[2] C. Favazza, J. Trice, A.K. Gangopadhyay, H. Garcia, R. Sureshkumar, and Ramki Kalyanaraman. Nanoparticle ordering by dewetting of co on $\mathrm{SiO}_{2}$. Journal of Electronic Materials, 35(8):1618-1620, 2006.

[3] C. Favazza, R. Kalyanaraman, and R. Sureshkumar. Robust nanopatterning by laser-induced dewetting of metal nanofilms. Nanotechnology, 17:4229, 2006.

[4] J. Trice, D. Thomas, C. Favazza, R. Sureshkumar, and R. Kalyanaraman. Pulsed-laser-induced dewetting in nanoscopic metal films: Theory and experiments. Physical Review B, 75(23):235439, 2007.

[5] P.D. Rack, Y.F. Guan, J.D. Fowlkes, A.V. Melechko, and M.L. Simpson. Pulsed laser dewetting of patterned thin metal films: A means of directed assembly. Applied Physics Letters, 92:223108, 2008.

[6] Joseph T. McKeown, Nicholas A. Roberts, Jason D. Fowlkes, Yueying Wu, Thomas LaGrange, Bryan W. Reed, Geoffrey H. Campbell, and Philip D. Rack. Real-time observation of nanosecond liquid-phase assembly of nickel nanoparticles via pulsed-laser heating. Langmuir, 28(49):17168-17175, 2012.

[7] Yoonseok Oh and Myeongkyu Lee. Single-pulse transformation of ag thin film into nanoparticles via laser-induced dewetting. Applied Surface Science, 399:555 - 564, 2017.

[8] Y.H. Lei, R.X. Yu, T. Shibayama, J. Ishioka, and S. Watanabe. Insitu observation of self-assembly of quasi-two-dimensional au nanosubmicron particles on -sic substrates via nanosecond-pulsed laser 
irradiation-induced dewetting of thin au films. Materials Letters, 164:202 - 205, 2016.

[9] V. S. Ajaev and D. A. Willis. Thermocapillary flow and rupture in films of molten metal on a substrate. Physics of Fluids, 15:3144, 2003.

[10] J. Trice, D. Thomas, C. Favazza, R. Sureshkumar, and R. Kalyanaraman. Novel Self-Organization Mechanism in Ultrathin Liquid Films: Theory and Experiment. Phys. Rev. Lett., 101:017802, 2008.

[11] L. Kondic, J. A. Diez, P. D. Rack, Y. Guan, and J. D. Fowlkes. Nanoparticle assembly via the dewetting of patterned thin metal lines: Understanding the instability mechanisms. Physical Review E, 79:026302, 2009 .

[12] J. D. Fowlkes, L. Kondic, J. Diez, Y. Wu, and P. D. Rack. Self-assembly versus directed assembly of nanoparticles via pulsed laser induced dewetting of patterned metal films. Nano Letters, 11(6):2478-2485, 2011.

[13] Y.-J. Oh, J.-H. Kim, C.V. Thompson, and C.A. Ross. Templated assembly of co-pt nanoparticles via thermal and laser-induced dewetting of bilayer metal films. Nanoscale, 5(1):401-407, 2013.

[14] J. D. Fowlkes, N. A. Roberts, Y. Wu, J. A. Diez, A. G. Gonzlez, C. Hartnett, K. Mahady, S. Afkhami, L. Kondic, and P. D. Rack. Hierarchical nanoparticle ensembles synthesized by liquid phase directed selfassembly. Nano Letters, 14(2):774-782, 2014.

[15] X. Li, Y. He, Y. Wang, J. Dong, and H. Li. Dewetting properties of metallic liquid film on nanopillared graphene. Scientific Reports, 4, 2014.

[16] K. Mahady, S. Afkhami, and L. Kondic. On the influence of initial geometry on the evolution of fluid filaments. Phys. Fluids, 27:092104, 2015 .

[17] C. A. Hartnett, K. Mahady, J. D. Fowlkes, S. Afkhami, L. Kondic, and P. D. Rack. Instability of nano- and microscale liquid metal filaments: Transition from single droplet collapse to multidroplet breakup. Langmuir, 31(50):13609-13617, 2015. 
[18] S. Yadavali, V.P. Sandireddy, and R. Kalyanaraman. Transformation of irregular shaped silver nanostructures into nanoparticles by under water pulsed laser melting. Nanotechnology, 27(19), 2016.

[19] L.-X. Lu, Y.-M. Wang, B.M. Srinivasan, M. Asbahi, J.K.W. Yang, and Y.-W. Zhang. Nanostructure formation by controlled dewetting on patterned substrates: A combined theoretical, modeling and experimental study. Scientific Reports, 6, 2016.

[20] Hendrik Reinhardt, Clemens Pietzonka, Bernd Harbrecht, and Norbert Hampp. Laser-directed self-organization and reaction control in complex systems: A facile synthesis route for functional materials. Advanced Materials Interfaces, 1(2):1300060-n/a, 2014.

[21] Hendrik M. Reinhardt, Kerstin Bücker, and Norbert A. Hampp. Directed assembly of gold nanowires on silicon via reorganization and simultaneous fusion of randomly distributed gold nanoparticles. Optics Express, 23(9):11965-11974, May 2015.

[22] A. Atena and M. Khenner. Thermocapillary effects in driven dewetting and self assembly of pulsed-laser-irradiated metallic films. Phys. Rev. B, 80:075402, Aug 2009.

[23] N. Dong and L. Kondic. Instability of nanometric fluid films on a thermally conductive substrate. Phys. Rev. Fluids, 1:063901, 2016.

[24] J.E. Kline and J.P. Leonard. Suppression of dewetting phenomena during excimer laser melting of thin metal films on $\mathrm{sio}_{2}$. Thin Solid Films, 488(1?2):306 - 313, 2005.

[25] A. G. González, J. A. Diez, Y. Wu, J. D. Fowlkes, P. D. Rack, and L. Kondic. Instability of liquid $\mathrm{Cu}$ films on a $\mathrm{SiO}_{2}$ substrate. Langmuir, 29:9378-9387, 2013.

[26] K. Kajihara, Y. Ikuta, M. Oto, M. Hirano, L. Skuja, and H. Hosono. Uv-vuv laser induced phenomena in sio2 glass. Nuclear Iinstruments and methods in physics research section B: Beam interactions with materials and atoms, 218:323 - 331, 2004.

[27] Jason D. Fowlkes, Lou Kondic, Javier A. Diez, Alejandro G. Gonzalez, Yueying Wu, Nick A. Roberts, Cliff E. McCold, and Philip D. Rack. 
Parallel assembly of particles and wires on substrates by dictating instability evolution in liquid metal films. Nanoscale, 4:7376-7382, 2012.

[28] Y. Wu, J. D. Fowlkes, N. A. Roberts, J. A. Diez, L. Kondic, A. G. Gonzlez, and P. D. Rack. Competing liquid phase instabilities during pulsed laser induced self-assembly of copper rings into ordered nanoparticle arrays on sio2. Langmuir, 27(21):13314-13323, 2011.

[29] D. W. Bäuerle. Laser Processing and Chemistry. Springer-Verlag Berlin Heidelberg, 4th edition, 2011.

[30] A. D. Rakić, A. B. Djurišić, J. M. Elazar, and M. L. Majewski. Optical properties of metallic films for vertical-cavity optoelectronic devices. Applied Optics, 37(22):5271-5283, 1998.

[31] C. E. Lesher and F. J. Spera. Chapter 5 - thermodynamic and transport properties of silicate melts and magma. In H. Sigurdsson, editor, The Encyclopedia of Volcanoes, pages 113 - 141. Academic Press, 2nd edition edition, 2015.

[32] V. Alexiades and A.D. Solomon. Mathematical modelling of freezing and melting processes. Hemisphere Publishing Corporation, 1st edition, 1993.

[33] J. M. Hill. One-Dimensional Stefan Problems: An Introduction. Longman Scientific \& Technical, 1st edition, 1987.

[34] S. L. Mitchell and M. Vynnycky. Finite-difference methods with increased accuracy and correct initialization for onedimensional stefan problems. Applied Mathematics and Computation, 215(4):1609-1621, 2009 .

[35] F. Font, T. G. Myers, and S. L. Mitchell. A mathematical model for nanoparticle melting with density change. Microfluidics and Nanofluidics, 18(2):233-243, 2015. 